\title{
Optimizing the Temporal Resolution of Fast-Scan Cyclic Voltammetry
}

\author{
Brian M. Kile, ${ }^{\dagger}$ Paul L. Walsh, ${ }^{\dagger}$ Zoé A. McElligott, ${ }^{\dagger}$ Elizabeth S. Bucher, ${ }^{\dagger}$ Thomas S. Guillot, ${ }^{\dagger}$
}

Ali Salahpour, ${ }^{\ddagger}$ Marc G. Caron, ${ }^{\S}$ and R. Mark Wightman ${ }^{*} \dagger$

${ }^{\dagger}$ Department of Chemistry and Neuroscience Center, University of North Carolina at Chapel Hill, Chapel Hill, North Carolina 27599, United States

${ }^{\ddagger}$ Department of Pharmacology and Toxicology, University of Toronto, Toronto, Ontario M5S 1A8, Canada

${ }^{\S}$ Department of Cell Biology, Duke University, Durham, North Carolina 27710, United States

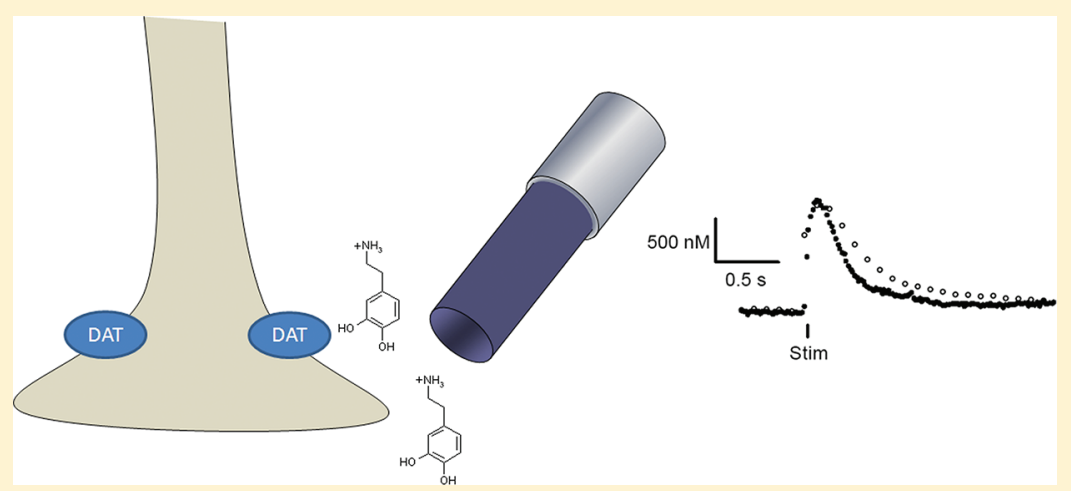

ABSTRACT: Electrochemical detection with carbon-fiber microelectrodes has become an established method to monitor directly the release of dopamine from neurons and its uptake by the dopamine transporter. With constant potential amperometry (CPA), the measured current provides a real time view of the rapid concentration changes, but the method lacks chemical identification of the monitored species and markedly increases the difficulty of signal calibration. Monitoring with fast-scan cyclic voltammetry (FSCV) allows species identification and concentration measurements but often exhibits a delayed response time due to the time-dependent adsorption/desorption of electroactive species at the electrode. We sought to improve the temporal resolution of FSCV to make it more comparable to CPA by increasing the waveform repetition rate from 10 to $60 \mathrm{~Hz}$ with uncoated carbon-fiber electrodes. The faster acquisition led to diminished time delays of the recordings that tracked more closely with CPA measurements. The measurements reveal that FSCV at $10 \mathrm{~Hz}$ underestimates the normal rate of dopamine uptake by about $18 \%$. However, FSCV collection at 10 and $60 \mathrm{~Hz}$ provide identical results when a dopamine transporter (DAT) blocker such as cocaine is bath applied. To verify further the utility of this method, we used transgenic mice that overexpress DAT. After accounting for the slight adsorption delay time, FSCV at $60 \mathrm{~Hz}$ adequately monitored the increased uptake rate that arose from overexpression of DAT and, again, was similar to CPA results. Furthermore, the utility of collecting data at $60 \mathrm{~Hz}$ was verified in an anesthetized rat by using a higher scan rate $(2400 \mathrm{~V} / \mathrm{s})$ to increase sensitivity and the overall signal.

KEYWORDS: Carbon-fiber microelectrode, dopamine, striatum, constant potential amperometry, dopamine uptake, Michaelis-Menten, dopamine transporter, cocaine

$\mathrm{T}$ he concentration of the neurotransmitter dopamine in brain extracellular fluid is regulated by the balance between release and uptake. Release of dopamine originates from vesicle exocytosis, ${ }^{1}$ while uptake is mediated by the dopamine transporter. ${ }^{2,3}$ Both release and uptake are rapid processes and control the subsecond fluctuations of dopamine concentrations observed in freely moving animals in brain regions such as the nucleus accumbens and dorsal striatum. ${ }^{4,5} \mathrm{~A}$ sensor must possess adequate temporal resolution in order to track the rapid concentration changes of dopamine that arise from these opposing processes. Carbon-fiber microelectrodes enable rapid measurement of neurotransmitters in brain tissue, and the methodologies for employing them have been improved over time. ${ }^{6-8}$ Due to the presence of interfering anions, many experiments have employed Nafion coatings to improve analyte selectivity. ${ }^{9}$ Transport through Nafion films requires several hundred milliseconds. ${ }^{10}$ To account for this, the time delay due to Nafion is measured in solution and its contribution was accounted for in the data obtained in brain tissue. ${ }^{11,12}$ This can be done by deconvolution of the response time from the data ${ }^{13}$ or by modeling the response and convoluting it with the experimentally measured response time

Received: November 30, 2011

Accepted: January 30, 2012

Published: January 30, 2012 

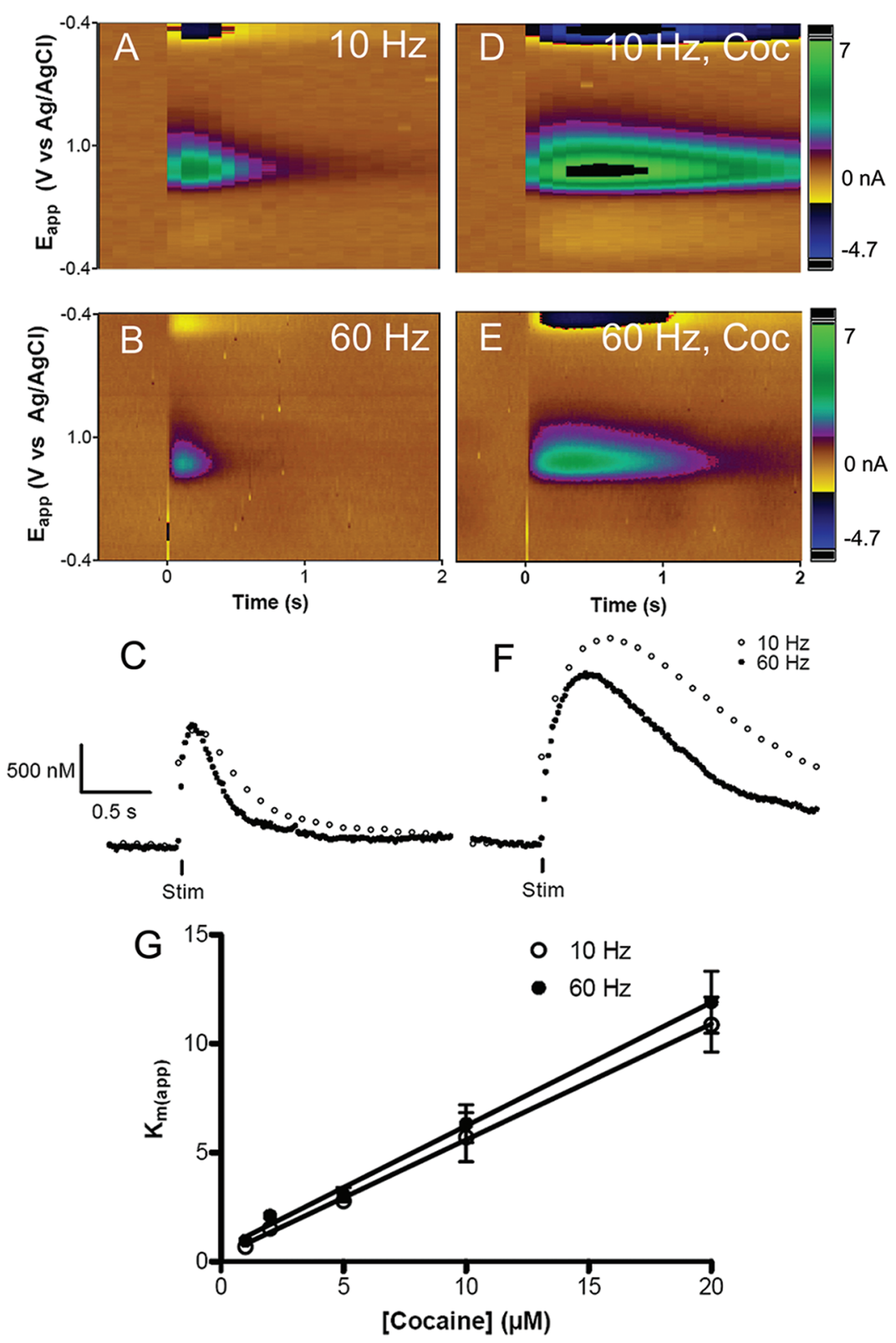

Figure 1. Dopamine release and uptake responses are dependent on the repetition rate of the cyclic voltammogram waveform. (A,B) Color plots of stimulated dopamine release recorded in the same location in a striatal brain slice with a waveform application frequency of (A) 10 and (B) $60 \mathrm{~Hz}$. Stimulation occurred at $t=0 \mathrm{~s}$. (C) Concentration traces for stimulated dopamine release extracted from the peak oxidation potential for dopamine at 10 and $60 \mathrm{~Hz}$. The dopamine concentration recorded at $10 \mathrm{~Hz}$ rises more slowly after stimulation and takes longer to return to baseline. Nonlinear regression accounting for the different adsorption times at the two different repletion rates reveals that the $10 \mathrm{~Hz}$ rate yields an underestimate of $V_{\max }$ by about $18 \% \pm 1 \%$. (D,E) Color plots of stimulated dopamine release recorded in the presence of $10 \mu \mathrm{M}$ cocaine collected at (D) 10 and (E) 60 Hz. (F) Concentration traces for stimulated dopamine release extracted from the peak oxidation potential for dopamine at 10 and $60 \mathrm{~Hz}$ in the presence of $10 \mu \mathrm{M}$ cocaine. Cocaine lowers the uptake rate and renders responses measured with 10 and $60 \mathrm{~Hz}$ repetition rates more similar. However, the signal recorded shows a $13 \% \pm 2 \%$ greater maximal dopamine concentration at $10 \mathrm{~Hz}$. $(\mathrm{G})$ Increasing concentrations of cocaine (1$20 \mu \mathrm{M}$ ) were used to generate a curve showing the change in $K_{\mathrm{m}}$ caused by cocaine. The $K_{\mathrm{i}}$ of cocaine was calculated from this curve and found to be the same within experimental error regardless of the waveform application frequency.

delay. ${ }^{14}$ In modeling dopamine release and uptake, release is considered to be instantaneous at each stimulus pulse and uptake is assumed to follow the Michaelis-Menten equation that is characterized by a maximal velocity $\left(V_{\max }\right)$ and the affinity of the transporter for the neurotransmitter $\left(K_{\mathrm{m}}\right)$, which is taken as the dopamine concentration when the rate of uptake is half of $V_{\max }$. For dopamine in the dorsal striatum of rodents, the $K_{\mathrm{m}}$ value is $\sim 0.2 \mu \mathrm{M}$ and the $V_{\max }$ is approximately $4 \mu \mathrm{M}$ / s. ${ }^{15,16}$ At low dopamine concentrations, uptake follows firstorder kinetics with a rate constant, $k$, given by $V_{\max } / K_{\mathrm{m}}$. In the rat dorsal striatum, $k$ values obtained following deconvolution of recordings at Nafion coated electrodes are $\sim 20 \mathrm{~s}^{-1}$, corresponding to a half-life of $\sim 40 \mathrm{~ms}$. Similar rate constants are found for dopamine uptake in the dorsal striatum of mice. ${ }^{17}$

When fast-scan cyclic voltammetry (FSCV) is used, the response to dopamine is not instantaneous, even with an uncoated carbon-fiber microelectrode. This is due to dopamine adsorbing to carbon surfaces in the time interval between cyclic voltammograms, and the kinetics of this adsorption/desorption process limit the time response. ${ }^{18}$ The adsorption process can 
be measured in a flow cell after use in brain tissue and then accounted for in the in vivo/ex vivo measurements by deconvolution or convolute-and-compare methods. Rate constants quite similar to those obtained with Nafion-coated electrodes are obtained with uncoated electrodes following appropriate handling of the response time delay caused by adsorption.

While the procedures for removing the response time delay seemed effective to determine rate constants from electrochemical recordings at the time that these procedures were developed, there was no way to independently confirm the values measured in intact tissue. Subsequently, Gonon and coworkers measured uptake rates with bare carbon-fiber microelectrodes by using constant potential amperometry (CPA). In CPA, molecules are immediately oxidized upon contact with the electrode, and adsorption does not slow the time response. When used to measure the disappearance of dopamine after stimulation, half-lives of $\sim 60 \mathrm{~ms}$ were obtained in the dorsal striatum, in reasonable agreement with the rates measured by FSCV once the convoluted response time delay was taken into account. ${ }^{19,20}$ In later work, our laboratory showed that the amperometric oxidation of dopamine in the presence of ascorbate could be used to obtain concentration calibrations due to the catalytic reaction between ascorbate and the dopamine-o-quinone. ${ }^{13}$ This work bridged the gap between the fast time response of $\mathrm{CPA}$ and the dopamine concentration measured by FSCV and thus provided a way to obtain Michaelis-Menten constants without deconvolution. Despite the documented advantages of CPA for measurement of dopamine uptake, this approach has disadvantages: the smaller currents are more affected by electrical noise, and the measurements lack the identifying analyte signatures that are provided by cyclic voltammograms. Therefore, a method that has the time resolution of CPA with the analyte identification of FSCV would be optimal for measuring fast electrochemical kinetics in biological systems.

To combine the advantages of CPA and FSCV, the time available for dopamine adsorption needs to be reduced. To accomplish this, we increased the FSCV repetition rate from 10 to $60 \mathrm{~Hz}$, which decreases the time the electrode is held at a negative potential and in turn the time available for adsorption to occur. ${ }^{18}$ To demonstrate the utility of the new approach, we compared it with the kinetics of dopamine release and uptake measured in brain slices with FSCV at a lower frequency, as well as CPA. The shorter pause between scans $(16.67 \mathrm{~ms}$ at 60 $\mathrm{Hz}$ versus $100 \mathrm{~ms}$ at $10 \mathrm{~Hz}$ ) resulted in faster electrode responses. A finite delay was still present, but once it was taken into account the kinetic values obtained were very similar to those measured by CPA. In further support of this assertion, the uptake rate in mice overexpressing the dopamine transporter (DAT-Tg) was also similar when measured by CPA or FSCV at $60 \mathrm{~Hz}$. Finally, scanning at $60 \mathrm{~Hz}$ was utilized in an anesthetized rat, keeping the overall shape of the signal, while increasing the scan rate in order to increase the sensitivity of the carbon fiber and the signal-to-noise ratio. ${ }^{21}$

\section{RESULTS AND DISCUSSION}

Temporal Response of Fast-Scan Cyclic Voltammetry. To examine how the sampling rate of FSCV affects measurements of dopamine dynamics in brain slices from wild-type (WT) animals, release and uptake evoked by a single stimulation pulse at the same location were compared when the waveform was applied at either 10 or $60 \mathrm{~Hz}$. Figure $1 \mathrm{~A}, \mathrm{~B}$ shows representative examples of color plots recorded during evoked dopamine release collected at $10 \mathrm{~Hz}$ (A) and $60 \mathrm{~Hz}$ (B). The data collected at $10 \mathrm{~Hz}$ appears more pixelated due to the lower collection frequency. Note that the maximal current recorded at $10 \mathrm{~Hz}$ is greater than that collected at $60 \mathrm{~Hz}$ due to the increased sensitivity with the longer time for adsorption. When the current at the peak potential for dopamine oxidation was converted to concentration for both collection rates, the maximal concentration of dopamine was the same. While both traces increased at the first measurement point after the stimulation pulse, the concentrations measured at $10 \mathrm{~Hz}$ took longer to return to baseline, a clear indication that the response time of the electrode has distorted the uptake time course (Figure 1C). ${ }^{18}$ With the $10 \mathrm{~Hz}$ repetition rate, the maximal response was $200 \mathrm{~ms}$ after the stimulus pulse, whereas it was 67 ms after the stimulus with the $60 \mathrm{~Hz}$ repetition frequency. This is because the $60 \mathrm{~Hz}$ sampling frequency diminishes the delay due to the kinetics of dopamine adsorption to the electrode.

To examine the reproducibility of these findings, they were repeated for a total of three to five stimulations for each recording frequency at an individual spot. This sequence was repeated in brain slices from five different animals. The maximal dopamine concentrations determined were $1.43 \pm$ $0.11 \mu \mathrm{M}$ when sampled at $10 \mathrm{~Hz}$ and $1.46 \pm 0.11 \mu \mathrm{M}$ when sampled at $60 \mathrm{~Hz}$, values that are not statistically different $(p>$ 0.05 , paired student's $t$ test, $n=5)$. Differences were seen, however, in the calculated $V_{\max }$ values when the two methods were compared. Following the convolute-and-compare regression procedure, the $V_{\max }$ values were significantly different $(p<$ $0.01, n=5$, paired student's $t$ test) when sampled at $10 \mathrm{~Hz}$ $(4.47 \pm 0.50 \mu \mathrm{M} / \mathrm{s})$ compared with $60 \mathrm{~Hz}$ sampling $(6.43 \pm$ $0.53 \mu \mathrm{M} / \mathrm{s}$ ) when all data from all animals was grouped. Note that DAT is heterogeneously expressed in the dorsal striatum, ${ }^{22}$ and therefore paired statistics were performed in order to compare the sampling rates in the same location. Across the five slices examined, the $V_{\max }$ value calculated from the $10 \mathrm{~Hz}$ data was $82 \% \pm 1 \%$ of the $V_{\max }$ calculated from the $60 \mathrm{~Hz}$ data. The lower value of $V_{\max }$ obtained with the $10 \mathrm{~Hz}$ sampling frequency was present despite accounting for the increased electrode delay time to reach $[\mathrm{DA}]_{\max }$ by the convolute-andcompare procedure, as well as the differences in electrode sensitivity.

Similar experiments were conducted in the presence of cocaine, a dopamine transporter blocker and uptake inhibitor. Figure 1D,E shows the color plots of the evoked dopamine release collected at (D) 10 and (E) $60 \mathrm{~Hz}$ in the presence of 10 $\mu \mathrm{M}$ cocaine. Dopamine concentrations determined were $13 \% \pm$ $2 \%$ greater when sampled at 10 rather than $60 \mathrm{~Hz}$ when $10 \mu \mathrm{M}$ cocaine was perfused onto the slice (Figure 1F). Since adsorption plays a significant role in the sensitivity of the carbon fiber to dopamine, the slower sampling rate gives more time for dopamine to diffuse from more remote terminals and adsorb to the electrode causing this discrepancy. ${ }^{23}$ This difference is not observed in the absence of cocaine because DAT is removing the excess dopamine from the extracellular space before it has time to diffuse to the electrode. Increases in stimulated peak dopamine concentration upon the application of cocaine have been previously reported using $10 \mathrm{~Hz}$ repetition rates. ${ }^{22}$ While these observations may have overestimated the increase in maximal concentration of dopamine due to the increased time for adsorption of dopamine from terminals not directly adjacent to the electrode, similar observations of an increase have been shown with ampero- 
metric recordings, which have a faster time response and do not include adsorption of dopamine to the electrode. ${ }^{20}$ When cocaine was present, $V_{\max }$ values determined from collecting FSCV data at 10 and $60 \mathrm{~Hz}$ were similar, indicating that when uptake is slower, measured rates are independent of acquisition frequency.

To quantify the similarities between the two methods when uptake is slowed, a complete dose-response curve for cocaine $(1-20 \mu \mathrm{M})$ was run at a single location in each brain slice using the two different repetition rates to obtain apparent $K_{\mathrm{m}}$ values for the concentration of cocaine. ${ }^{24}$ The apparent value of $K_{\mathrm{m}}$ $\left(K_{\mathrm{m}, \mathrm{app}}\right)$ after drug administration was determined by fixing the predrug $V_{\max }$ value for a recording location and obtaining $K_{\mathrm{m} \text {,app }}$ by simplex regression. The dissociation constant for a competitive inhibitor $\left(K_{\mathrm{i}}\right)$ can be found by linear regression using the expression $K_{\mathrm{m} \text {,app }}=K_{\mathrm{m}}\left(1+[\mathrm{I}] / K_{\mathrm{i}}\right)$, where $[\mathrm{I}]$ is the inhibitor concentration, and the slope of $K_{\mathrm{m} \text {,app }}$ versus [I] is $K_{\mathrm{m}} / K_{\mathrm{i}}{ }^{25}$ The slope when cyclic voltammograms were sampled at $10 \mathrm{~Hz}$ was $0.57 \pm 0.06$, and it was $0.60 \pm 0.04$ when they were sampled at $60 \mathrm{~Hz}$, values that are not significantly different $(p>0.05$, paired student's $t$ test, $n=5)$. These experiments yielded $K_{\mathrm{i}}$ values for cocaine of $0.36 \pm 0.04 \mu \mathrm{M}$ for the $10 \mathrm{~Hz}$ data and $0.34 \pm 0.02 \mu \mathrm{M}$ at $60 \mathrm{~Hz}$, values in close agreement with previous reports with data collected at $10 \mathrm{~Hz}^{26}$ or $60 \mathrm{~Hz}^{25}$

The experiments employing FSCV at a higher repetition rate $(60 \mathrm{~Hz})$ show that previous measurements at $10 \mathrm{~Hz}$ slightly underestimated $V_{\max }$ values by about $18 \%$. This is because the adsorption/desorption rates are more pronounced with longer times between voltage scans. This masks the rate of dopamine uptake to a greater degree than with data collection at $60 \mathrm{~Hz}$ (Figure 1C). Slowing dopamine uptake with cocaine reduces the differences between measured uptake rates obtained at the two FSCV sampling frequencies because the time delay caused by adsorption becomes insignificant compared with the reduced rate of uptake (Figure $1 \mathrm{~F}$ ). This should also be true for kinetic determinations in brain regions where dopamine uptake is slower such as the basolateral amygdaloid nucleus, ${ }^{22}$ because the temporal distortion obtained with lower sampling can be more accurately taken into account when uptake rates are slow. Furthermore, $10 \mathrm{~Hz}$ would also be preferable for studying the uptake of norepinephrine in the bed nucleus of the stria terminalis (BNST) for a number of reasons. Uptake of norepinephrine is slower in the BNST, carbon fiber microelectrodes are less sensitive to norepinephrine than dopamine, and the total amount of norepinephrine is smaller; ${ }^{27}$ thus $10 \mathrm{~Hz}$ gives larger signals from the increased time allowed for adsorption.

Temporal Response of Fast-Scan Cyclic Voltammetry Compared with Amperometry. It is necessary when performing CPA in a brain slice to add ascorbate to the aCSF buffer at a concentration mimicking in vivo brain levels $(500 \mu \mathrm{M})$. The ascorbate participates in a catalytic cycle with dopamine-o-quinone that maintains the dopamine diffusion layer close to the electrode and thus allows an accurate in vitro calibration of the amperometric electrode. ${ }^{28}$ The dopamine signal recorded during stimulated release was enhanced in the presence of ascorbate by nearly 2 -fold $(79 \% \pm 0.09 \%$ increase, $p<0.001$, paired student's $t$ test, $n=5$, Figure 2) as a result of the catalytic reaction. It did not, however, alter the time of the peak response or the measured half-life of the signal to return to baseline. Since calibration is not meaningful for the amperometric trace without ascorbate, measurements of the

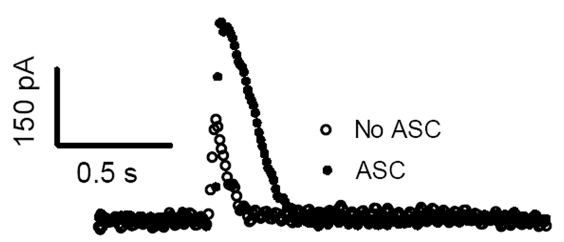

Figure 2. Addition of ascorbate to striatal brain slices increases amperometric signals. The addition of $500 \mu \mathrm{M}$ ascorbate to the aCSF perfusing the slice increases the dopamine signal measured by amperometry due to recycling of dopamine-o-quinone caused by the catalytic oxidation of ascorbate by dopamine-o-quinone.

descending slopes of traces made with and without ascorbate were assessed in current units. The maximal uptake slope without ascorbate present was $-1.26 \pm 0.08 \mathrm{nA} / \mathrm{s}(n=5)$, while it was $-1.11 \pm 0.05 \mathrm{nA} / \mathrm{s}(n=5)$ when ascorbate was present, values that are not significantly different $(p>0.05$, paired student's $t$ test).

FSCV data collected at $60 \mathrm{~Hz}$ was compared with CPA by using both methods at the same location in a brain slice with $500 \mu \mathrm{M}$ ascorbate present (Figure 3). The time to reach peak

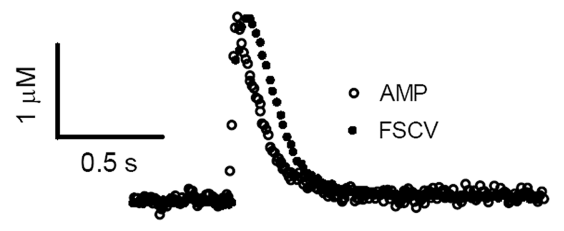

Figure 3. Stimulated dopamine release monitored with fast-scan cyclic voltammetry repeated at $60 \mathrm{~Hz}$ and amperometry. Both measurements were in the presence of $500 \mu \mathrm{M}$ ascorbate. A time delay due to adsorption can be seen in the fast-scan cyclic voltammetric response indicated by a longer time to reach a maximum and a slower decay to baseline. The adsorptive delay with cyclic voltammetry was accounted for using the convolute-and-compare method to obtain rate constants.

dopamine concentration was $40 \mathrm{~ms}$ with FSCV repeated at 60 $\mathrm{Hz}$ (Figure 3). In contrast, the time was $<10 \mathrm{~ms}$ with CPA (Figures 2 and 3 ), a time delay that we have previously attributed to diffusion from the release site to the electrode. ${ }^{28}$ The slower response of the FSCV traces is a clear indication of the adsorption/desorption kinetics; however, when these kinetics are accounted for by deconvolution of the adsorption delay, regression of the CPA signals and FSCV traces collected at $60 \mathrm{~Hz}$ yielded similar $V_{\max }$ values of $6.43 \pm 0.53$ and $6.48 \pm$ $0.40 \mu \mathrm{M} / \mathrm{s}$, respectively ( $p>0.05$, paired student's $t$ test, $n=5)$. These values are statistically indistinguishable and confirm that with a $60 \mathrm{~Hz}$ repetition rate, FSCV is as accurate as CPA for measuring dopamine uptake kinetics when the adsorptive response time delay is handled appropriately with the convolution-and-compare method. The addition of ascorbate did not change the cyclic voltammetric signal amplitude or kinetics (data not shown).

These data directly compare FSCV at $60 \mathrm{~Hz}$ with CPA in units of dopamine concentration; CPA and FSCV were used on brain slices with $500 \mu \mathrm{M}$ ascorbate in the aCSF buffer. The addition of ascorbate allows calibration of the amperometric electrode signal in dopamine concentration units as well as an increase in the amplitude of the signal. ${ }^{13}$ However, the rate of dopamine uptake measured by CPA is identical with or without ascorbate despite the increased amplitude with ascorbate addition. Ascorbate does not affect the measured rate of 

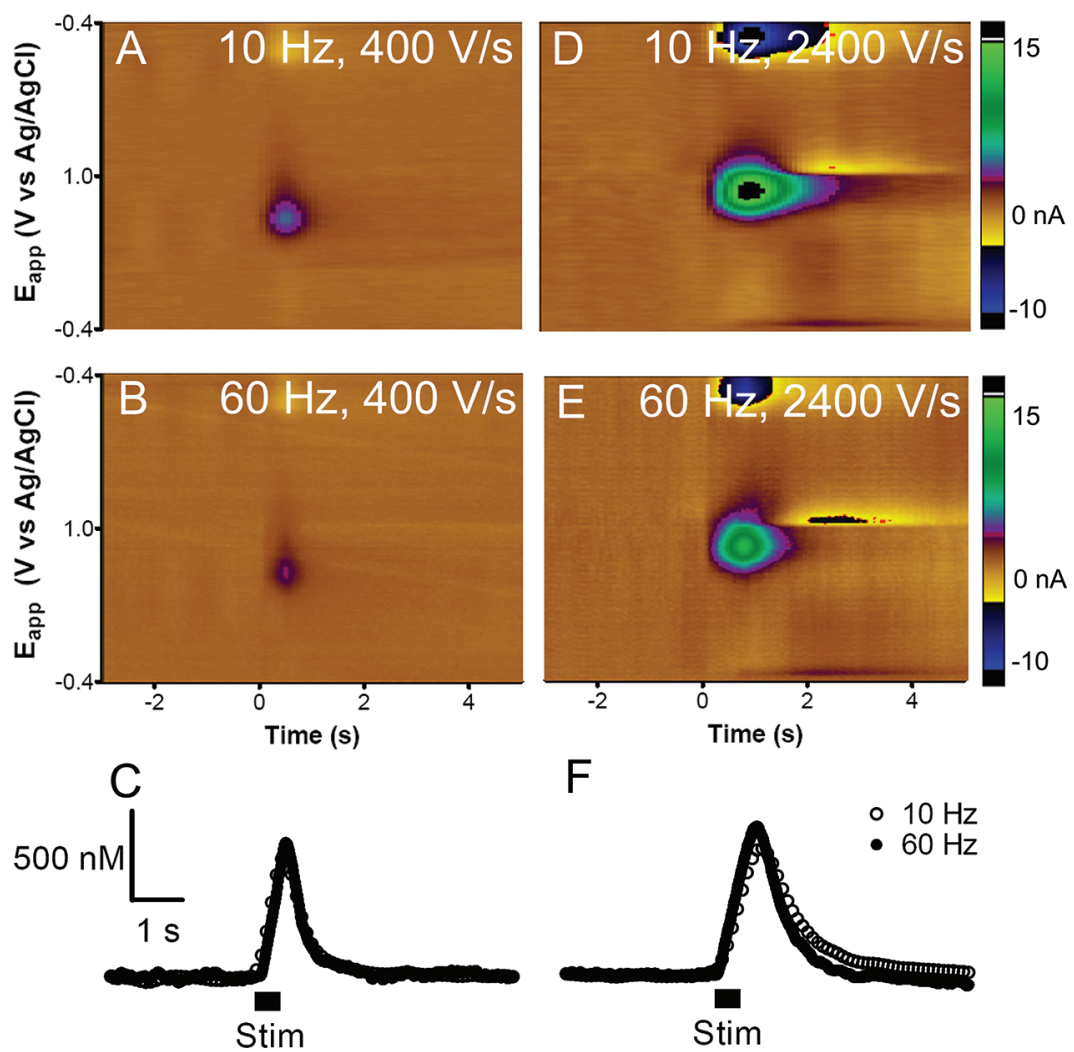

Figure 4. Stimulated dopamine release can be monitored in an anesthetized rat at $60 \mathrm{~Hz}$. (A,B) Color plots of stimulated dopamine release collected using $400 \mathrm{~V} / \mathrm{s}$ as the scan rate collected at (A) 10 and (B) $60 \mathrm{~Hz}$. (C) Concentration versus time traces for the data collected at 10 and $60 \mathrm{~Hz}$ from the peak oxidation potential for dopamine using a $400 \mathrm{~V} / \mathrm{s}$ scan rate. The concentrations calculated at 10 and $60 \mathrm{~Hz}$ are not different from each other. (D,E) Color plots of stimulated dopamine release collected using a scan rate of $2400 \mathrm{~V} / \mathrm{s}$ collected at (A) 10 and (B) $60 \mathrm{~Hz}$. The peak current for dopamine oxidation is about 5.5 times greater than obtained at $400 \mathrm{~V} / \mathrm{s}$. (F) Concentration versus time traces for the data collected at 10 and 60 $\mathrm{Hz}$ from the peak oxidation potential for dopamine using a $2400 \mathrm{~V} / \mathrm{s}$ scan rate. Switching from 10 to $60 \mathrm{~Hz}$ at this higher scan rate does not dramatically decrease the signal-to-noise ratio as it does at $400 \mathrm{~V} / \mathrm{s}$, while maintaining the same calculated peak concentration of dopamine.

dopamine disappearance because DAT removes dopamine at a faster rate than the electrode consumes it.

Measurement of Dopamine at $60 \mathrm{~Hz}$ in an Anesthetized Rat. To determine the utility of applying the voltammetric waveform at $60 \mathrm{~Hz}$ in vivo, dopamine release was evoked in the striatum of an anesthetized rat by stimulating the VTA. Figure $4 A, B$ shows the color plots for the dopamine release collected at (A) $10 \mathrm{~Hz}$ and (B) $60 \mathrm{~Hz}$ using $400 \mathrm{~V} / \mathrm{s}$ as the scan rate. When the electrode was scanned at $10 \mathrm{~Hz}$, the current was $1.6 \pm 0.1$ times larger than that when the collection frequency was $60 \mathrm{~Hz}(n=5)$. Note, however, that when converted to concentration, the shape of the release is the same as are the measured concentrations (Figure 4C, $p>0.05$, paired student's $t$ test, $n=5$ ). The smaller observed signal decreases the signal-to-noise ratio.

As previously reported, faster scan rates for scanning to $1.0 \mathrm{~V}$ increases the sensitivity of the electrode to dopamine in the anesthetized rat brain. ${ }^{21}$ Here, faster scan rates $(2400 \mathrm{~V} / \mathrm{s})$ were used to maintain the signal-to-noise ratio when the waveform was applied at $60 \mathrm{~Hz}$ during an anesthetized surgery. This helps to increase the signal in two ways. First, for adsorbing species, it increases the voltammetric peak current proportional to the scan rate applied to the electrode. ${ }^{29}$ Also, it increases the amount of time between scans for adsorption from 9.7 to $15.5 \mathrm{~ms}$ when collecting data at $60 \mathrm{~Hz}$. Figure 4D,E shows color plots for the dopamine release collected at (D) 10 $\mathrm{Hz}$ and (E) $60 \mathrm{~Hz}$ using $2400 \mathrm{~V} / \mathrm{s}$ as the scan rate. The peak oxidation current is $5.5 \pm 0.1$ times larger at $2400 \mathrm{~V} / \mathrm{s}$ compared with the data collected using $400 \mathrm{~V} / \mathrm{s}$ at both 10 and $60 \mathrm{~Hz}$ ( $n=5$ for each). While the current measured at $10 \mathrm{~Hz}$ is $1.4 \pm 0.1$ times larger than that collected at $60 \mathrm{~Hz}$, postcalibration showed that the peak concentrations measured were not significantly different between the two application frequencies at the higher scan rate (Figure 4F, $p>0.05$, paired student's $t$ test, $n=5$ ). These observations indicate that the use of faster scan rates and an increased frequency does not evoke release from nearby terminals, as was previously observed for striatal cell firing rates, ${ }^{21}$ and the shape of the release and uptake profile is unchanged. These data indicate that FSCV can be used at $60 \mathrm{~Hz}$ in the anesthetized preparation if an increase in temporal resolution is desired, as long as the scan rate is increased to maintain the signal-to-noise ratio.

Measurement of Uptake Rates in Transgenic Mice Using Fast Scan Cyclic Voltammetry. The ability of FSCV at $60 \mathrm{~Hz}$ to measure uptake rates that are even faster than those found in WT animals was evaluated in brain slices from mice that overexpress the dopamine transporter (DAT-Tg mice). The DAT-Tg animal has four additional copies of the DAT gene leading to a greater amount of DAT expression on the membrane of dopamine neurons compared with WT animals. ${ }^{30}$ Although these animals have four additional copies of the DAT, the amount of functional transporter in the brains of these mice is $\sim 50 \%$ higher than normal. ${ }^{22}$ The dopamine uptake rate in 
brain slices containing the dorsal striatum of transgenic mice was measured using both FSCV at $60 \mathrm{~Hz}$ and CPA.

When release was evoked by single pulse stimulation in tissue from WT animals, amperometric recordings gave an almost immediate rise in dopamine followed by decay to baseline (Figure 5A). In striatal tissue from transgenic animals, the

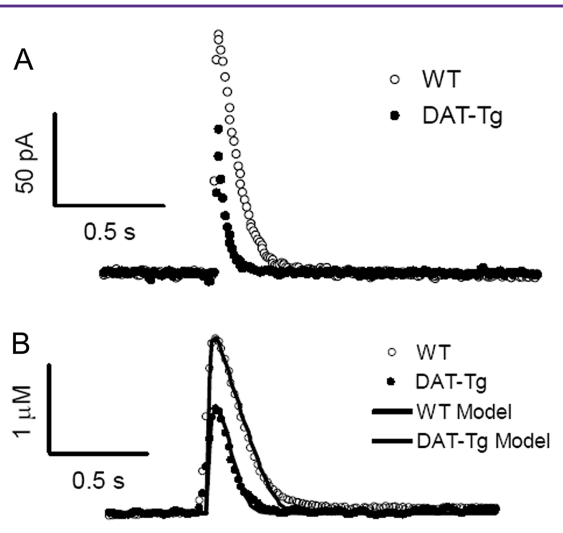

Figure 5. Transgenic mice overexpressing DAT have faster striatal dopamine uptake. (A) Representative dopamine release and uptake measured in WT and DAT-Tg mice with amperometry. The dopamine release profile from DAT-Tg animals is smaller in magnitude and has a faster decay than the profile from WT animals. The ratio of $V_{\max }$ values taken from both genotypes gives a DAT-Tg/WT ratio of $1.66 \pm 0.36$, a significant enhancement ( $p<0.01, n=4$ each genotype). (B) Representative release profiles from WT and DAT-Tg animals measured with fast-scan cyclic voltammetry are qualitatively similar to their amperometric counterparts. Deconvolution and simplex modeling (solid lines) of these traces gave $V_{\max }$ values of $5.0 \mu \mathrm{M} / \mathrm{s}$ for the WT animal and $7.2 \mu \mathrm{M} / \mathrm{s}$ for the DAT-Tg animal.

amplitude of release was smaller and the decay faster than those in WT tissue. The initial descending slope was used to calculate the $V_{\max }$ value of uptake. Ascorbate was not used in these experiments, so to compare CPA with FSCV results, the values are expressed as the initial uptake slope ratio of DAT-Tg to WT ( $n=5$ animals of each genotype, both measurements at single sites). This ratio of the initial amperometric slopes from uptake was found to be $1.62 \pm 0.36$.

Dopamine release and uptake was also evaluated with FSCV at $60 \mathrm{~Hz}$ at the same locations as the CPA measurements using an identical stimulation. Release measured by FSCV was lower in DAT-Tg striatal brain slices than in WT striatal brain slices (Figure 5B). The $V_{\max }$ values were determined by regression analysis accounting for the delay time due to adsorption, and the predicted curves overlay the data very well (Figure 5B, solid traces). The $V_{\max }$ values obtained from the single example traces in Figure $5 \mathrm{~B}$ were $5.0 \mu \mathrm{M} / \mathrm{s}$ for WT and $7.2 \mu \mathrm{M} / \mathrm{s}$ for DAT-Tg. We have previously reported that the $V_{\max }$ of DAT$\mathrm{Tg}$ is $160 \%$ of that measured in WT animals using FSCV sampled at $60 \mathrm{~Hz}(n=4$, both genotypes $){ }^{30}$ statistically identical to that seen by CPA $(p>0.05$, student's $t$ test $)$. The similarity in the $V_{\max }$ ratio between WT and DAT-Tg given by $\mathrm{CPA}(1.62 \pm 0.36)$ and FSCV at $60 \mathrm{~Hz}(1.61 \pm 0.04)$ confirms that the latter method has sufficient temporal resolution to monitor uptake in animals overexpressing the dopamine transporter when the reduced response time delay due to adsorption is taken into account.

Our results show that the method used to handle the response time delay inherent to FSCV at $10 \mathrm{~Hz}$ is suitable if the rate of uptake is sufficiently slow, such as in the presence of cocaine. Conditions of faster biological kinetics, however, require an increased sampling rate that improves data quality in two ways: first, it decreases the response time delay caused by adsorption, and second, it provides a greater sampling density to describe the uptake process. When uptake has a half-life of $\sim 100 \mathrm{~ms}$ or less, sampling at $10 \mathrm{~Hz}$ does not provide enough measurement points for accurate characterization of dopamine's disappearance. A 6-fold increase in sampling rate both increases the sampling density and decreases the time for the adsorption response, the net result of which is a more accurate determination of uptake kinetics. Applying the voltammetric waveform at $60 \mathrm{~Hz}$ yielded rate constants for dopamine uptake that were quite similar to those measured by $\mathrm{CPA},{ }^{20}$ a method that does not exhibit a response time delay. ${ }^{13}$ Furthermore, we were able to perform experiments in anesthetized animals at not only higher frequencies but higher scan rates, allowing for increased sensitivity of the electrode to the analyte. As previously pointed out, ${ }^{19}$ for characterization of rapid kinetics, CPA with its lack of response time delay is clearly superior, but two clear advantages of FSCV over CPA are ready identification of the analyte and that the measured concentrations are independent of ascorbate. Our data clearly illustrate that increasing the repetition rate of FSCV decreases the response time delay caused by adsorption and, after accounting for the minimal remaining adsorption time, yields kinetic measurements identical to CPA.

\section{METHODS}

Animals. Wild-type (WT) mice (C57BL/6J) used in this study were purchased from Jackson Laboratory (Bar Harbor, ME). Male Sprague-Dawley rats were purchased from Charles River Laboratories (Wilmington, MA). Animals were housed and cared for according to the animal use protocols determined by the Institute for Animal Care at the University of North Carolina at Chapel Hill. Dopamine transporter transgenic (DAT-Tg) mice were created as previously described $^{30}$ and housed according to protocol set by the Institute for Animal Care at Duke University.

Electrochemistry. Cylindrical carbon-fiber $(6 \mu \mathrm{m}$ diameter, Thornel T-650, Amoco, Greenville, SC) microelectrodes were fabricated as described previously by sealing in a glass capillary. ${ }^{18}$ The length of the protruding fiber was $50 \mu \mathrm{m}$ for slice experiments and $100 \mu \mathrm{m}$ for anesthetized experiments. Waveform application, current monitoring, and stimulus application were all controlled by locally written software (Tarheel CV, Labview) through a home-built potentiostat (UEI, UNC electronics shop), using a 6052E (National Instruments, Austin, TX) DAC-ADC card and a 6711 (National Instruments, Austin, TX) as the timing card. For fast-scan cyclic voltammetry (FSCV), the carbon-fiber microelectrode was held at $-0.40 \mathrm{~V}$ vs $\mathrm{Ag} / \mathrm{AgCl}$ between voltage scans. A triangular waveform $(-0.40$ to $1.00 \mathrm{~V}$ to $-0.40 \mathrm{~V}$, scan rate of $600 \mathrm{~V} / \mathrm{s}$ for slices and 400 or $2400 \mathrm{~V} / \mathrm{s}$ for anesthetized experiments) was applied with a repetition rate of 10 or $60 \mathrm{~Hz}$. For experiments utilizing $2400 \mathrm{~V} / \mathrm{s}$ as the scan rate, analog background subtraction, as described previously, was used to prevent saturation of the DAC card. ${ }^{31}$ Data are displayed as color plots, which show time on the ordinate, applied potential on the abscissa, and current displayed in false color. ${ }^{32}$ The current at the peak oxidation potential for dopamine $(0.6 \mathrm{~V}$ vs $\mathrm{Ag} / \mathrm{AgCl})$ was used to evaluate dopamine concentration changes with time. For constant potential amperometry (CPA), the current was digitized for $1 \mathrm{~ms}$ at a repetition frequency of $120 \mathrm{~Hz}$, and the electrode was held at $0.70 \mathrm{~V}$ vs $\mathrm{Ag} / \mathrm{AgCl}$, which oxidizes dopamine and is optimal for the dopamine regenerating reaction of dopamine-o-quinone with ascorbate.

Brain Slice Experiments. Slice experiments were performed as previously described with minor modifications. ${ }^{33}$ Briefly, mice were anesthetized with ether and quickly decapitated, and the brain was rapidly removed and placed in an ice cold, oxygenated $\left(95 \% \mathrm{O}_{2} / 5 \%\right.$ $\mathrm{CO}_{2}$ ) artificial cerebral spinal fluid (aCSF) solution (in mM, 20 
HEPES, $2.4 \mathrm{CaCl}_{2}, 1.2 \mathrm{MgCl}_{2}, 1.2 \mathrm{NaH}_{2} \mathrm{PO}_{4}, 2.45 \mathrm{KCl}, 126 \mathrm{NaCl}, 11$ glucose, and $25 \mathrm{NaHCO}_{3}$ ) where $\mathrm{pH}$ was adjusted to 7.4. Coronal brain slices $(300 \mu \mathrm{m})$ containing the dorsal striatum were prepared using a Lancer Vibratome (World Precision Instruments, Sarasota, $\mathrm{FL}$ ). Slices were superfused in oxygenated aCSF at $37{ }^{\circ} \mathrm{C}$ for $35-40$ min prior to recording. Slices were supplemented with $500 \mu \mathrm{M}$ ascorbate in some cases to enhance the amperometric signal and facilitate electrode calibration. ${ }^{13}$ FSCV and CPA experiments were conducted at the same locations in a slice because the $V_{\max }$ varies with location, presumably due to the variable distribution of the transporter. ${ }^{30}$ Experiments were performed on a microscope (Nikon Fn-1, Gibraltar Stage) fitted with a slice perfusion chamber (RC-22, Warner Instruments, Hamden CT).

Neurotransmitter release was evoked by local electrical stimulation delivered with a tungsten bipolar stimulating electrode placed on the surface of the slice (Frederick Haer Co., Bowdoinham, ME). The stimulation consisted of a single computer-generated, biphasic $(2 \mathrm{~ms}$ per phase), constant current $(350 \mu \mathrm{A})$ pulse. The current pulse was optically isolated from the preparation (NL 800, Neurolog, Medical Systems, Great Neck, NY). Stimulations were separated by at least 5 min to ensure stable release that was unaffected by prior stimulations. ${ }^{26}$ Neurotransmitter release was detected with a carbonfiber microelectrode placed $75-100 \mu \mathrm{m}$ into the slice at a distance of $100-200 \mu \mathrm{m}$ from the stimulating electrode. Electrode placements were made with the aid of the microscope.

Surgical Procedures. For in vivo measurements, male SpragueDawley rats $(275-350 \mathrm{~g})$ were anesthetized with urethane $(1.5 \mathrm{mg}$ / $\mathrm{kg}$ ) and placed in a stereotaxic frame. Placement of the electrodes was performed using a brain atlas with coordinates given relative to bregma. ${ }^{34}$ Holes were drilled into the skull to allow for the placement of the carbon-fiber microelectrode in the dorsal striatum $(+1.3 \mathrm{~A} / \mathrm{P}$, $+2.3 \mathrm{M} / \mathrm{L},-4.0$ to $-5.0 \mathrm{D} / \mathrm{V}$, all coordinates are in $\mathrm{mm})$, and the bipolar tungsten stimulating electrode (Plastics One, Roanoke, VA) in the ventral tegmental area (VTA) $(-5.2 \mathrm{~A} / \mathrm{P},+1.0 \mathrm{M} / \mathrm{L},-7.0$ to -9.0 $\mathrm{D} / \mathrm{V})$. The $\mathrm{Ag} / \mathrm{AgCl}$ reference electrode was placed in the contralateral hemisphere from the working and stimulating electrodes. Trains of biphasic pulses were applied to the stimulating electrode (2 ms per phase, $24 \mathrm{p}, 60 \mathrm{~Hz}, 300 \mu \mathrm{A})$. Data were collected at 10 and 60 $\mathrm{Hz}$ using scan rates of 400 and $2400 \mathrm{~V} / \mathrm{s}$.

Determination of Kinetic Parameters. Nonlinear regression using a simplex algorithm was used to extract kinetic constants from the FSCV data for dopamine uptake and to remove the contribution of the response time delay. ${ }^{35}$ Dopamine concentrations were assumed to immediately rise with the stimulation and then fall to baseline following Michaelis-Menten kinetics. ${ }^{11}$ Initial estimates are made of the amount released, $[\mathrm{DA}]_{\max }$ and maximum uptake velocity, $V_{\max }$. The $K_{\mathrm{m}}$ value was fixed at $0.2 \mu \mathrm{M}$, the value found in both rats ${ }^{36}$ and mice. ${ }^{17}$ Since deconvolution may result in spurious results, we used the convolute-and-compare approach. ${ }^{14}$ The calculated curves were convoluted with the measured response time, and the nonlinear regression then adjusted $[\mathrm{DA}]_{\max }$ and $V_{\max }$ until a best fit was obtained as judged by minimization of the residual. The electrode time constants were electrode specific and were between 15 and $50 \mathrm{~ms}$ for a $60 \mathrm{~Hz}$ sampling rate and between 100 and $200 \mathrm{~ms}$ with the $10 \mathrm{~Hz}$ sampling rate. A time constant of $0 \mathrm{~s}$ was used for CPA measurements in the presence of ascorbate.

Two methods were used to find $V_{\max }$ from the amperometric data. First, the slope of the descending portion of the curve from $[\mathrm{DA}]_{\max }$ to $1 / 2[\mathrm{DA}]_{\max }$ was measured directly, and the slope of this line was taken to be the $V_{\max }$ of uptake for that location. Alternatively, the trace was fitted using the simplex modeling program with the electrode time constant set to a value of zero. Both approaches provided comparable $V_{\max }$ values of uptake.

Calibration of Electrodes. After use in a brain slice, electrodes were calibrated for the response to dopamine in a flow injection apparatus. Because the amplitude and rise time of the dopamine response depends on the repetition frequency, measurements were made with both the 10 and $60 \mathrm{~Hz}$ frequencies. Response times were determined by the $10-90 \%$ rise time. Unless noted, amperometry experiments were conducted with ascorbic acid $(500 \mu \mathrm{M})$ added both to the slice and to calibration buffers to enable calibration of the electrode. ${ }^{13}$

Data Analysis. Data are shown as mean \pm SEM. Student's $t$ tests were performed using Prism 4.0 (GraphPad, San Diego, CA). Statistical significance is determined by $p$ values less than 0.05 , and $n=$ number of animals unless otherwise indicated.

\section{AUTHOR INFORMATION}

\section{Corresponding Author}

*Mailing address: UNC-CH Chemistry Department, CB \#3290, University of North Carolina at Chapel Hill, Chapel Hill, NC 27599. E-mail: rmw@unc.edu. Phone: (919) 9621472.

\section{Author Contributions}

Brian Kile and Paul Walsh planned the experiments, performed experiments, and wrote the paper. Zoe McElligott, Beth Bucher, and Thomas Guillot performed experiments and wrote the paper. Ali Salahpour and Marc Caron provided DAT-Tg animals and edited the paper. R. Mark Wightman planned experiments and wrote the paper.

\section{Notes}

The authors declare no competing financial interest.

\section{ACKNOWLEDGMENTS}

This research was supported in part by grants from the NIH (Grant NS38879 to R.M.W. and Grant MH-73358 to M.G.C.).

\section{ABBREVIATIONS}

FSCV, fast-scan cyclic voltammetry; CPA, constant potential amperometry; DAT, dopamine transporter; DAT-Tg, DAT transgenic; VTA, ventral tegmental area

\section{REFERENCES}

(1) Sulzer, D., and Pothos, E. N. (2000) Regulation of quantal size by presynaptic mechanisms. Rev. Neurosci. 11 (2-3), 159-212.

(2) Latini, S., and Pedata, F. (2001) Adenosine in the central nervous system: Release mechanisms and extracellular concentrations. J. Neurochem. 79 (3), 463-484.

(3) Giros, B., Jaber, M., Jones, S. R., Wightman, R. M., and Caron, M. G. (1996) Hyperlocomotion and indifference to cocaine and amphetamine in mice lacking the dopamine transporter. Nature 379, 606-612.

(4) Sombers, L. A., Beyene, M., Carelli, R. M., and Wightman, R. M. (2009) Synaptic overflow of dopamine in the nucleus accumbens arises from neuronal activity in the ventral tegmental area. J. Neurosci. 29 (6), 1735-1742.

(5) Robinson, D. L., Howard, E. C., McConnell, S., Gonzales, R. A., and Wightman, R. M. (2009) Disparity between tonic and phasic ethanol-induced dopamine increases in the nucleus accumbens of rats. Alcohol.: Clin. Exp. Res. 33 (7), 1187-1196.

(6) Gonon, F., Buda, M., Cespuglio, R., Jouvet, M., and Pujol, J. F. (1980) In vivo electrochemical detection of catechols in the neostriatum of anesthetized rats: Dopamine or DOPAC? Nature 286, 902-904.

(7) Ewing, A. G., Dayton, M. A., and Wightman, R. M. (1981) Pulse voltammetry with microvoltammetric electrodes. Anal. Chem. 53, $1842-1847$.

(8) Armstrong-James, M., and Millar, J. (1979) Carbon fibre microelectrodes. J. Neurosci. Methods 1 (3), 279-287.

(9) Gerhardt, G. A., Oke, A. F., Nagy, G., Moghaddam, B., and Adams, R. N. (1984) Nafion-coated electrodes with high selectivity for CNS electrochemistry. Brain Res. 290, 390-395.

(10) Kristensen, E. W., Kuhr, W. G., and Wightman, R. M. (1987) Temporal characterization of perfluorinated ion exchange coated 
microvoltammeteric electrodes for in vivo use. Anal. Chem. 59, 17521757.

(11) Wightman, R. M., Amatore, C., Engstrom, R. C., Hale, P. D., Kristensen, E. W., Kuhr, W. G., and May, L. J. (1988) Real-time characterization of dopamine overflow and uptake in the rat striatum. Neuroscience 25 (2), 513-523.

(12) Kawagoe, K. T., and Wightman, R. M. (1994) Characterization of amperometry for in vivo measurement of dopamine dynamics in the rat brain. Talanta 41 (6), 865-74.

(13) Venton, B. J., Troyer, K. P., and Wightman, R. M. (2002) Response times of carbon fiber microelectrodes to dynamic changes in catecholamine concentration. Anal. Chem. 74 (3), 539-546.

(14) Engstrom, R. C., Wightman, R. M., and Kristensen, E. W. (1988) Diffusional distortion in monitoring dynamic events. Anal. Chem. 60, 652-656.

(15) Horn, A. S. (1990) Dopamine uptake: A review of progress in the last decade. Prog. Neurobiol. 34 (5), 387-400.

(16) Bunin, M. A., and Wightman, R. M. (1998) Measuring uptake rates in intact tissue. Methods Enzymol. 296, 689-707.

(17) John, C. E., and Jones, S. R. (2007) Voltammetric characterization of the effect of monoamine uptake inhibitors and releasers on dopamine and serotonin uptake in mouse caudate-putamen and substantia nigra slices. Neuropharmacology 52 (8), 1596-1605.

(18) Bath, B. D., Michael, D. J., Trafton, B. J., Joseph, J. D., Runnels, P. L., and Wightman, R. M. (2000) Subsecond adsorption and desorption of dopamine at carbon-fiber microelectrodes. Anal. Chem. 72 (24), 5994-6002.

(19) Dugast, C., Suaud-Chagny, M. F., and Gonon, F. (1994) Continuous in vivo monitoring of evoked dopamine release in the rat nucleus accumbens by amperometry. Neuroscience 62, 647-654.

(20) Suaud-Chagny, M. F., Dugast, C., Chergui, K., Msghina, M., and Gonon, F. (1995) Uptake of dopamine released by impulse flow in the rat mesolimbic and striatal systems in vivo. J. Neurochem. 65, 26032611.

(21) Keithley, R. B., Takmakov, P., Bucher, E. S., Belle, A. M., Owesson-White, C. A., Park, J., and Wightman, R. M. (2011) Higher sensitivity dopamine measurements with faster-scan cyclic voltammetry. Anal. Chem. 83 (9), 3563-3571.

(22) Jones, S. R., Garris, P. A., Kilts, C. D., and Wightman, R. M. (1995) Comparison of dopamine uptake in the basolateral amygdaloid nucleus, caudate-putamen, and nucleus accumbens of the rat. $J$. Neurochem. 64 (6), 2581-2589.

(23) Bath, B. D., Michael, D. J., Trafton, B. J., Joseph, J. D., Runnels, P. L., and Wightman, R. M. (2000) Subsecond adsorption and desorption of dopamine at carbon-fiber microelectrodes. Anal. Chem. 72 (24), 5994-6002.

(24) Duncan, R. R., Greaves, J., Wiegand, U. K., Matskevich, I., Bodammer, G., Apps, D. K., Shipston, M. J., and Chow, R. H. (2003) Functional and spatial segregation of secretory vesicle pools according to vesicle age. Nature 422 (6928), 176-180.

(25) Johnson, M. A., Rajan, V., Miller, C. E., and Wightman, R. M. (2006) Dopamine release is severely compromised in the R6/2 mouse model of Huntington's disease. J Neurochem 97 (3), 737-746.

(26) Jones, S. R., Garris, P. A., and Wightman, R. M. (1995) Different effects of cocaine and nomifensine on dopamine uptake in the Caudate-Putamen and nucleus accumbens. J. Pharmacol. Exp. Ther. 274, 396-403.

(27) Park, J., Kile, B. M., and Wightman, R. M. (2009) In vivo voltammetric monitoring of norepinephrine release in the rat ventral bed nucleus of the stria terminalis and anteroventral thalamic nucleus. Eur. J. Neurosci. 30 (11), 2121-2133.

(28) Venton, B. J., Zhang, H., Garris, P. A., Phillips, P. E., Sulzer, D., and Wightman, R. M. (2003) Real-time decoding of dopamine concentration changes in the caudate-putamen during tonic and phasic firing. J. Neurochem. 87 (5), 1284-1295.

(29) Bard, A. J., and Faulkner, L. R. (2001) Electrochemical Methods: Fundamentals and Applications, 2nd ed., John Wiley \& Sons, Inc., New York.
(30) Salahpour, A., Ramsey, A. J., Medvedev, I. O., Kile, B., Sotnikova, T. D., Holmstrand, E., Ghisi, V., Nicholls, P. J., Wong, L., Murphy, K., Sesack, S. R., Wightman, R. M., Gainetdinov, R. R., and Caron, M. G. (2008) Increased amphetamine-induced hyperactivity and reward in mice overexpressing the dopamine transporter. Proc. Natl. Acad. Sci. U.S.A. 105 (11), 4405-4410.

(31) Hermans, A., Keithley, R. B., Kita, J. M., Sombers, L. A., and Wightman, R. M. (2008) Dopamine detection with fast-scan cyclic voltammetry used with analog background subtraction. Anal. Chem. 80 (11), 4040-4048.

(32) Michael, D., Travis, E. R., and Wightman, R. M. (1998) Color images for fast-scan CV measurements in biological systems. Anal. Chem. 70 (17), 586A-592A.

(33) Kile, B. M., Guillot, T. S., Venton, B. J., Wetsel, W. C., Augustine, G. J., and Wightman, R. M. (2010) Synapsins differentially control dopamine and serotonin release. J. Neurosci. 30 (29), 97629770 .

(34) Paxinos, G., Watson, C. (2007) The Rat Brain in Stereotaxic Coordinates, 6th ed., Elselvier, Amsterdam, The Netherlands.

(35) Wu, Q. Reith, M. E., Wightman, R. M., Kawagoe, K. T., and Garris, P. A. (2001) Determination of release and uptake parameters from electrically evoked dopamine dynamics measured by real-time voltammetry. J. Neurosci. Methods 112 (2), 119-133.

(36) Near, J. A., Bigelow, J. C., and Wightman, R. M. (1988) Comparison of uptake of dopamine in rat striatal chopped tissue and synaptosomes. J. Pharmacol. Exp. Ther. 245 (3), 921-927. 